九州大学学術情報リポジトリ

Kyushu University Institutional Repository

\title{
Spherical designs from norm-3 shell of integral lattices
}

Shigezumi, Junichi

Graduate School of Mathematics, Kyushu University: Student(D3): Algebraic Combinatorics

http://hdl. handle. net/2324/14234

出版情報：Asian-European Journal of Mathematics. 2 (2)，pp.239-253，2009-06 バージョン：

権利関係 : 


\title{
SPHERICAL DESIGNS FROM NORM-3 SHELL OF INTEGRAL LATTICES
}

\author{
JUNICHI SHIGEZUMI
}

Graduate School of Mathematics Kyushu University

Hakozaki 6-10-1 Higashi-ku, Fukuoka, 812-8581 Japan

E-mail address : j.shigezumi@math.kyushu-u.ac.jp

\begin{abstract}
A set of vectors all of which have a constant (non-zero) norm value in an Euclidean lattice is called a shell of the lattice. Venkov classified strongly perfect lattices of minimum 3 (Réseaux et "designs" sphérique, 2001), whose minimal shell is a spherical 5 -design. This note considers the classification of integral lattices whose shells of norm 3 are 5-designs.
\end{abstract}

Key Words and Phrases. spherical design, Euclidean lattice.

2000 Mathematics Subject Classification. Primary 05B30; Secondary 03G10.

\section{INTRODUCTION}

Let $L$ be an Euclidean lattice, which is a discrete vector space over $\mathbb{Z}$. The squared norm of a vector of the lattice is called the norm of the vector. Then, the set $s_{m}(L)$ of vectors of the lattice $L$ which take the same value $m$ for their norm is called the shell of the lattice, i.e. $s_{m}(L):=\{x \in L ;(x, x)=m\}$. Moreover, the shell of minimum $\min _{x \in L \backslash\{0\}}(x, x)$ of the lattice $L$ is called the minimal shell, which is denoted by $S(L)$.

Definition 0.1 (Spherical design [3]). Let $X$ be a non-empty finite set on the Euclidean sphere $S^{d-1}$, and let $t$ be a positive integer. $X$ is called a spherical $t$-design if

$$
\frac{1}{\left|S^{d-1}\right|} \int_{S^{d-1}} f(\xi) d \xi=\frac{1}{|X|} \sum_{\xi \in X} f(\xi)
$$

for every polynomial $f(x)=f\left(x_{1}, \ldots, x_{d}\right)$ of degree at most $t$.

For every nonempty shell $s_{m}(L)$ of a lattice $L$, a normalization $X=\frac{1}{\sqrt{m}} s_{m}(L)$ is considered, where $X$ is a finite set on an Euclidean sphere. A lattice, whose minimal shell is a spherical 4-design (i.e. a 5-design), is said to be strongly perfect.

B. B. Venkov proved the following theorem:

Theorem 1 (Venkov [9], Theorem 7.4). The strongly perfect lattices that are integral and of minimum 3 are $O_{1}, O_{7}, O_{16}, O_{22}$, and $O_{23}$. Furthermore, the minimal shell is a spherical 7-design only for the case of the lattice $\mathrm{O}_{23}$.

Now, as an expansion of the above theorem, we prove the following theorem:

Theorem 2. Let $L$ be an integral lattice. If its shell of norm 3 is a spherical 5 -design, then $L$ is isometric to one of the following nine lattices:

(1) $\mathbb{Z}^{7}$, whose minimum is equal to one.

(2) $\Lambda_{16,2,1}, \Lambda_{16,2,2}$ and $\Lambda_{16,2,3}$, whose minima are equal to two.

(3) $\mathrm{O}_{1}, \mathrm{O}_{7}, \mathrm{O}_{16}, \mathrm{O}_{22}$, and $\mathrm{O}_{23}$, whose minima are equal to three.

The definitions of the lattices in the above theorems are given in the next section. The remaining sections are devoted to the proof of Theorem 2 . 


\section{Definitions of the LAtTices in Theorem 2}

\subsection{Lattices of minimum 3.}

Lemma 1.1 (Venkov [9], Lemma 7.1). Let $L$ be an even integral lattice of dimension $n \geq 2$ and of minimum 4, and let e be a minimal vector of L. Denote by $p$ the orthogonal projection on the hyperplane $H=e^{\perp}$, put $L_{e}{ }^{\prime}=\{x \in L \mid(e, x) \equiv 0(\bmod 2)\}$, and let $L_{e}=p\left(L_{e}{ }^{\prime}\right)$. Suppose that one of the following two assumptions holds:

(1) There is $x \in L$ such that $(e, x) \equiv 1(\bmod 2)$;

(2) We have $(y, e) \equiv 0(\bmod 2)$ for all $y \in L$, and $L$ contains a vector $x$ such that $(e, x) \equiv 2(\bmod 4)$.

Then, $L_{e}$ is a odd integral lattice of minimum at least 3 , and we have $\operatorname{det}\left(L_{e}\right)=\operatorname{det}(L)$ under assumption (1) and $\operatorname{det}\left(L_{e}\right)=\frac{1}{4} \operatorname{det}(L)$ under assumption (2).

We denote by $\Lambda_{n}$ the laminated lattices for $2 \leq n \leq 24$ (See Conway-Sloane [2], Ch. 6). Note that $\Lambda_{n}$ is isometric to $\sqrt{2} \mathbb{E}_{n}$ for $n=6,7,8$, that $\Lambda_{16}$ is isometric to the Barnes-Wall lattice $B W_{16}$, and that $\Lambda_{24}$ is the Leech lattice. Then, we set $O_{1}=\sqrt{3} \mathbb{Z}$. We denote by $O_{7}$ (resp. $O_{23}$ ) the projected $\mathrm{E}_{e}$ associated with the laminated lattice $\Lambda_{8}\left(\right.$ resp. $\left.\Lambda_{24}\right)$. Finally, we denote by $O_{22}$ (resp. $O_{16}$ ) the orthogonal of $O_{1}$ (resp. $\mathrm{O}_{7}$ ) in $\mathrm{O}_{23}$.

We have $\operatorname{det}\left(O_{1}\right)=\operatorname{det}\left(O_{22}\right)=3, \operatorname{det}\left(O_{7}\right)=\operatorname{det}\left(O_{16}\right)=64$, and $\operatorname{det}\left(O_{23}\right)=1$; thus $O_{23}$ is unimodular.

The theta series of each lattice have the following form:

$$
\begin{aligned}
\Theta_{O_{1}} & =1+2 q^{3}+2 q^{12}+2 q^{27}+2 q^{48}+\cdots \\
\Theta_{O_{7}} & =1+56 q^{3}+126 q^{4}+576 q^{7}+756 q^{8}+1512 q^{11}+2072 q^{12} \\
& +4032 q^{15}+4158 q^{16}+5544 q^{19}+7560 q^{20}+12096 q^{23}+11592 q^{24}+\cdots \\
\Theta_{O_{16}} & =1+512 q^{3}+4320 q^{4}+18432 q^{5}+61440 q^{6}+193536 q^{7} \\
& +522720 q^{8}+1126400 q^{9}+2211840 q^{10}+4584960 q^{11}+8960640 q^{12}+\cdots \\
\Theta_{O_{22}} & =1+2816 q^{3}+49896 q^{4}+456192 q^{5}+2821632 q^{6}+13229568 q^{7} \\
& +50332590 q^{8}+163175936 q^{9}+467596800 q^{10}+1214196480 q^{11} \\
& +2900976144 q^{12}+\cdots \\
\Theta_{O_{23}} & =1+4600 q^{3}+93150 q^{4}+953856 q^{5}+6476800 q^{6}+32788800 q^{7} \\
& +133204500 q^{8}+458086400 q^{9}+1384998912 q^{10}+3771829800 q^{11} \\
& +9403968600 q^{12}+\cdots
\end{aligned}
$$

\begin{tabular}{|c|c|c|c|c|c|c|c|c|}
\hline & \multicolumn{4}{|c|}{$O_{7}$} & \multicolumn{4}{|c|}{$O_{16}$} \\
\hline$m$ & $d$ & $n$ & $s$ & $t$ & $d$ & $n$ & $s$ & $t$ \\
\hline 3 & 7 & 56 & 3 & 5 & 16 & 512 & 4 & 5 \\
\hline 4 & 7 & 126 & 4 & 5 & 16 & 4320 & 6 & 7 \\
\hline 5 & & & & & 16 & 18432 & 8 & 5 \\
\hline 6 & & & & & 16 & 61440 & 10 & 7 \\
\hline 7 & 7 & 576 & 7 & 5 & 16 & 193536 & 12 & 5 \\
\hline 8 & 7 & 756 & 8 & 53 & 16 & 522720 & 14 & 7 \\
\hline 9 & & & & & & & & \\
\hline 10 & & & & & & & & \\
\hline 11 & 7 & 1512 & 11 & 5 & & & & \\
\hline 12 & 7 & 2072 & 12 & 5 & & & & \\
\hline
\end{tabular}

Since $O_{23}$ is unimodular, we also have that $\Theta_{O_{23}}=\theta_{3}^{23}-46 \theta_{3}^{15} \Delta_{8}$, where $\Delta_{8}=\frac{1}{16} \theta_{2}^{4} \theta_{4}^{4}$ and $\theta_{i}$ for $i=2,3,4$ are known as Jacobi's theta functions. (See [2], [6])

Let $X$ be a nonempty finite set on the Euclidean sphere $S^{d-1}\left(\subset \mathbb{R}^{d}\right)$. We denote the distance set of $X$ by $A(X):=\{(x, y) ; x, y \in X, x \neq y\}$; then we call $X$ an $s$-distance set if $|A(X)|=s$. Now, $X$ is said to be a $(d, n, s, t)$-configuration if $X \subset S^{d-1}$ is of order $n(:=|X|)$, a $s$-distance set, and a spherical $t$-design. The following table contains the $(d, n, s, t)$-configuration of each shell of norm $m$ of the lattice: 


\begin{tabular}{|c|c|c|c|c|c|c|c|c|}
\hline & \multicolumn{4}{|c|}{$O_{22}$} & \multicolumn{4}{|c|}{$O_{23}$} \\
\hline$m$ & $d$ & $n$ & $s$ & $t$ & $d$ & $n$ & $s$ & $t$ \\
\hline 3 & 22 & 2816 & 4 & 5 & 23 & 4600 & 4 & 7 \\
\hline 4 & 22 & 49896 & 6 & 5 & 23 & 93150 & 6 & 7 \\
\hline 5 & 22 & 456192 & 8 & 5 & 23 & 953856 & 8 & 7 \\
\hline
\end{tabular}

1.2. Lattices of minimum 2. Let $\varepsilon_{1}, \ldots, \varepsilon_{16}$ be an orthonormal basis of $\mathbb{R}^{16}$. We denote some vectors

$$
\begin{gathered}
f_{1}:=\frac{\varepsilon_{1}+\cdots+\varepsilon_{8}}{2}+\varepsilon_{9}, \quad f_{2}:=\varepsilon_{1}+\frac{\varepsilon_{9}+\cdots+\varepsilon_{16}}{2}, \\
f_{3}:=\varepsilon_{1}+\varepsilon_{5}+\varepsilon_{9}+\varepsilon_{13}, \\
f_{4}:=\varepsilon_{1}+\varepsilon_{3}+\varepsilon_{5}+\varepsilon_{7}, \quad f_{5}:=\varepsilon_{1}+\varepsilon_{3}+\varepsilon_{9}+\varepsilon_{11}, \quad f_{6}:=\varepsilon_{1}+\varepsilon_{3}+\varepsilon_{13}+\varepsilon_{15} .
\end{gathered}
$$

Now, we define the following three lattices

$$
\begin{aligned}
& \Lambda_{16,2,1}:=\left\langle\left(A_{1}\right)^{16}, f_{1}, f_{2}, f_{3}, f_{4}, f_{5}, f_{6}\right\rangle, \\
& \Lambda_{16,2,2}:=\left\langle\left(D_{4}\right)^{4}, f_{1}, f_{2}, f_{3}\right\rangle, \\
& \Lambda_{16,2,3}:=\left\langle\left(D_{8}\right)^{2}, f_{1}, f_{2}\right\rangle,
\end{aligned}
$$

where we put root systems in the above definitions as $\left(A_{1}\right)^{16}:=\left\{ \pm\left(\varepsilon_{2 i-1} \pm \varepsilon_{2 i}\right) ; 1 \leqslant i \leqslant 8\right\},\left(D_{4}\right)^{4}:=$ $\left\{ \pm\left(\varepsilon_{i} \pm \varepsilon_{j}\right) ; 1 \leqslant i<j \leqslant 4,5 \leqslant i<j \leqslant 8,9 \leqslant i<j \leqslant 12\right.$, or $\left.13 \leqslant i<j \leqslant 16\right\}$, and $\left(D_{8}\right)^{2}:=$ $\left\{ \pm\left(\varepsilon_{i} \pm \varepsilon_{j}\right) ; 1 \leqslant i<j \leqslant 8\right.$ or $\left.9 \leqslant i<j \leqslant 16\right\}$. Then, we have $\left(A_{1}\right)^{16} \subset\left(D_{4}\right)^{4} \subset\left(D_{8}\right)^{2}$ and $\Lambda_{16,2,1} \subset \Lambda_{16,2,2} \subset \Lambda_{16,2,3}$. Furthermore, we have

$$
\Lambda_{16,2,3}=\Lambda_{16,2,2} \cup\left(\varepsilon_{1}+\varepsilon_{5}+\Lambda_{16,2,2}\right) \quad \text { and } \quad \Lambda_{16,2,2}=\Lambda_{16,2,1} \cup\left(\varepsilon_{1}+\varepsilon_{3}+\Lambda_{16,2,1}\right) .
$$

Remark 1.1. We denote some other vectors

$$
\begin{aligned}
f_{7} & :=\varepsilon_{1}+\varepsilon_{2}+\varepsilon_{3}+\varepsilon_{4}, \quad f_{8}:=\varepsilon_{1}+\varepsilon_{2}+\varepsilon_{5}+\varepsilon_{6}, \quad f_{9}:=\varepsilon_{1}+\varepsilon_{2}+\varepsilon_{7}+\varepsilon_{8}, \\
f_{10} & :=\varepsilon_{1}+\varepsilon_{2}+\varepsilon_{9}+\varepsilon_{10}, \quad f_{11}:=\varepsilon_{1}+\varepsilon_{2}+\varepsilon_{11}+\varepsilon_{12}, \quad f_{12}:=\varepsilon_{1}+\varepsilon_{2}+\varepsilon_{13}+\varepsilon_{14}, \\
f_{13} & :=\varepsilon_{1}+\varepsilon_{2}+\varepsilon_{15}+\varepsilon_{16} .
\end{aligned}
$$

Then, we can write

$$
O_{16}=\left\langle\left(\sqrt{2} A_{1}\right)^{16}, f_{1}, f_{2}, f_{3}, f_{4}, f_{5}, f_{6}, f_{7}, f_{8}, f_{9}, f_{10}, f_{11}, f_{12}, f_{13}\right\rangle
$$

and we have $\left(\sqrt{2} A_{1}\right)^{16}:=\left\{ \pm 2 \varepsilon_{i} ; 1 \leqslant i \leqslant 16\right\} \subset\left(A_{1}\right)^{16}$ and $O_{16} \subset \Lambda_{16,2,1}$. Furthermore, we have

$$
\Lambda_{16,2,1}=O_{16} \cup\left(\varepsilon_{1}+\varepsilon_{2}+O_{16}\right) .
$$

We have $\operatorname{det}\left(\Lambda_{16,2,1}\right)=16, \operatorname{det}\left(\Lambda_{16,2,2}\right)=4$, and $\operatorname{det}\left(\Lambda_{16,2,3}\right)=1$; thus $\Lambda_{16,2,3}$ is unimodular. We obtain the theta series of the lattices by numerical calculation as the following form:

$$
\begin{aligned}
\Theta_{\Lambda_{16,2,1}} & =1+32 q^{2}+1024 q^{3}+8160 q^{4}+36864 q^{5}+127360 q^{6}+387072 q^{7} \\
& +1016288 q^{8}+2252800 q^{9}+4564416 q^{10}+9169920 q^{11}+17395328 q^{12}+\cdots \\
\Theta_{\Lambda_{16,2,2}} & =1+96 q^{2}+2048 q^{3}+15840 q^{4}+73728 q^{5}+259200 q^{6}+774144 q^{7} \\
& +2003424 q^{8}+4505600 q^{9}+9269568 q^{10}+18339840 q^{11}+34264704 q^{12}+\cdots \\
\Theta_{\Lambda_{16,2,3}} & =1+224 q^{2}+4096 q^{3}+31200 q^{4}+147456 q^{5}+522880 q^{6}+1548288 q^{7} \\
& +3977696 q^{8}+9011200 q^{9}+18679872 q^{10}+36679680 q^{11}+68003456 q^{12}+\cdots
\end{aligned}
$$

Since $\Lambda_{16,2,3}$ is unimodular, we also have that $\Theta_{\Lambda_{16,2,3}}=\theta_{3}^{16}-32 \theta_{3}^{8} \Delta_{8}$.

The following table is the $(d, n, s, t)$-configuration of each shell of norm $m$ of the lattice: 


\begin{tabular}{cccccc} 
& \multicolumn{5}{c}{$\Lambda_{16,2,1}$} \\
\cline { 1 - 3 } \cline { 5 - 6 }$\frac{m}{2}$ & & $d$ & $n$ & $s$ & $t$ \\
3 & & 16 & 1024 & 6 & 5 \\
4 & & 16 & 8160 & 8 & 3 \\
5 & & 16 & 36864 & 10 & 5 \\
6 & & 16 & 127360 & 12 & 3 \\
7 & & 16 & 387072 & 14 & 5 \\
8 & & 16 & 1016288 & 16 & 3 \\
9 & & 16 & 2252800 & 18 & 5 \\
\hline
\end{tabular}

\begin{tabular}{cccc}
\multicolumn{4}{c}{$\Lambda_{16,2,2}$} \\
\hline$d$ & $n$ & $s$ & $t$ \\
\hline 16 & 96 & 4 & 3 \\
16 & 2048 & 6 & 5 \\
16 & 15840 & 8 & 3 \\
16 & 73728 & 10 & 5 \\
16 & 259200 & 12 & 3 \\
16 & 774144 & 14 & 5 \\
16 & 2003424 & 16 & 3 \\
\hline
\end{tabular}

\begin{tabular}{cccc}
\multicolumn{4}{c}{$\Lambda_{16,2,3}$} \\
\hline$d$ & $n$ & $s$ & $t$ \\
\hline 16 & 224 & 4 & 3 \\
16 & 4096 & 6 & 5 \\
16 & 31200 & 8 & 3 \\
16 & 147456 & 10 & 5 \\
16 & 522880 & 12 & 3 \\
16 & 1548288 & 14 & 5 \\
\hline
\end{tabular}

1.3. Lattice of minimum 1. We have $\operatorname{det}\left(\mathbb{Z}^{7}\right)=1$, thus $\mathbb{Z}^{7}$ is unimodular.

The theta series of the lattices have the following form:

$$
\begin{aligned}
\Theta_{\mathbb{Z}^{7}} & =1+14 q+84 q^{2}+280 q^{3}+574 q^{4}+840 q^{5}+1288 q^{6}+2368 q^{7} \\
& +3444 q^{8}+3542 q^{9}+4424 q^{10}+7560 q^{11}+9240 q^{12}+\cdots
\end{aligned}
$$

Since $\mathbb{Z}^{7}$ is unimodular, we also have $\Theta_{\mathbb{Z}^{7}}=\theta_{3}^{7}$.

For the spherical design from each shell of $\mathbb{Z}^{7}$, the following facts are already known:

Theorem 1.2 (Pache [7], parts of Theorem 25 and Proposition 26).

(1) For $n \geqslant 2$, all the nonempty shells of $\mathbb{Z}^{n}$ are spherical 3-designs.

(2) The following shells are spherical 5-designs:

$$
\begin{array}{ll}
s_{m}\left(\mathbb{Z}^{4}\right) & m=2 a, \quad a \geqslant 1 . \\
s_{m}\left(\mathbb{Z}^{7}\right) & m=4^{a}(8 b+3), \quad a, b \geqslant 0 .
\end{array}
$$

(3) For $n \geqslant 2$ and $1 \leqslant m \leqslant 1200$, the nonempty shells of norm $m$ of $\mathbb{Z}^{n}$ are not spherical 5-designs, except for the above cases.

Remark 1.2. $\mathbb{Z}^{7}$ and $O_{7}$ have $8\left(\begin{array}{l}7 \\ 3\end{array}\right)=280$ and $8 \cdot 7=56$ vectors of norm 3 , respectively. Then, as a natural question, can we write $s_{3}\left(\mathbb{Z}^{7}\right)$ as a disjoint union of configurations isometric to $s_{3}\left(O_{7}\right)$ ? The answer is no.

There are 30 subsets of $s_{3}\left(\mathbb{Z}^{7}\right)$ which are isometric to $s_{3}\left(O_{7}\right)$. However, any 3 such subsets are not disjoint. Here, we can choose 2 disjoint subsets, for example, $( \pm 1, \pm 1,0, \pm 1,0,0,0)^{C}$ and $( \pm 1,0, \pm 1, \pm 1,0,0,0)^{C}$, where " \pm " indicates that we take all possible sign changes, and $C$ indicates that we take any cyclic shifts.

Remark 1.3. Note that all the lattices in this section are 3-lattices, which are generated by some vectors of norm 3 .

Let $L$ be an integral lattice of dimension $n$, whose shell of norm 3 is a spherical 5 -design. By $X:=s_{3}(L)$ the shell of norm 3 is denoted. The argument of Theorem 2 (3) is just equivalent to Theorem 1 . Thus, we may suppose $\min (L)$ equal to 1 or 2 .

\section{ON SPHERICAL DESIGNS}

Theorem 2.1 (Venkov [9], Theorem 3.1). Let $X \in S^{n-1}$ be a finite set, and $t$ be a positive integer. By $e$ (resp. o ) the greatest even (resp. odd) integer which is at most $t$ is denoted. Then, $X$ is a spherical $t$-design if and only if there is a constant $c_{e}$ such that, for every $\alpha \in \mathbb{R}^{n}$, we have the two equations

$$
\sum_{x \in X}(x, \alpha)^{e}=c_{e}(\alpha, \alpha)^{e / 2} \quad \text { and } \quad \sum_{x \in X}(x, \alpha)^{o}=0 .
$$

If the above two equations hold, by repetition of the Laplacian $\Delta_{\alpha}$, we always have the formulae

$$
\sum_{x \in X}(x, \alpha)^{k}=c_{k}(\alpha, \alpha)^{k / 2} \text { and } \sum_{x \in X}(x, \alpha)^{l}=0
$$

for any even $k \leq e$ and any odd $l \leq o$, where the notation $\Delta_{y}$ refers to derivation with respect to the variable $y$. We also have

$$
c_{k}=\frac{1 \cdot 3 \cdot 5 \cdots(k-1)}{n(n+2) \cdots(n+k-2)}|X| .
$$


In this paper, the finite sets on spheres from shells of Euclidean lattices are considered. Then, every set is antipodal, thus the second equation always holds. Also, 5-designs from vectors of norm 3 are also considered here. Thus it is necessary and sufficient to consider the following two equations:

$$
\begin{aligned}
& \sum_{x \in X}(x, \alpha)^{2}=\frac{3|X|}{n}(\alpha, \alpha), \\
& \sum_{x \in X}(x, \alpha)^{4}=\frac{27|X|}{n(n+2)}(\alpha, \alpha)^{2} .
\end{aligned}
$$

Again, let $X$ be the shell of norm 3 of the lattice $L$. For any vectors $x_{0} \in X$, we denote $n_{i}:=\mid\{x \in$ $\left.X ;\left(x_{0}, x\right)=i\right\} \mid$ for $i=0,1,2$. By the above equations, taking $\alpha=x_{0}$, we have

$$
n_{0}=\frac{4 n^{2}-37 n+153}{4 n(n+2)}|X|-20, \quad n_{1}=\frac{3(4 n-19)}{2 n(n+2)}|X|+15, \quad n_{2}=\frac{3(25-n)}{8 n(n+2)}|X|-6 .
$$

Note that these results do not depend on the choice of $x_{0}$.

\section{Minimum of Lattices}

Let $t \in S(L)$ be a minimal vector of the lattice $L$. Since $(x \pm t, x \pm t)-(t, t)=(x, x) \pm 2(x, t) \geq 0$ for any $x \in X$, we have $|(x, t)| \leqslant \frac{1}{2}(x, x)=\frac{3}{2}$ (cf. [9], Lemma 6.10). Thus, we have

$$
(x, t) \in\{0, \pm 1\} \text {. }
$$

Then, we denote $p_{i}:=|\{x \in X ;(x, t)=i\}|$ for $i=0,1$. By the equalities (8) and (9), taking $\alpha=t$, we have

$$
(t, t)=\frac{n+2}{9}, \quad p_{0}=\frac{2(n-1)}{3 n}|X|, \quad p_{1}=\frac{n+2}{6 n}|X|
$$

(cf. [9], Lemma 7.11)

If $\min (L)=1$, by the first equation, the dimension of the lattice $L$ is equal to 7 . By [8], there are only two 3-lattices whose shells of norm 3 are spherical 5 -designs, which are $O_{7}$ and $\mathbb{Z}^{7}$. Here, a 3-lattice is an integral lattice which is generated by vectors of norm 3 . If we consider the lattice $L^{\prime}$ which is generated by $s_{3}(L)$, then $L^{\prime}$ is a 3 -lattice such that $s_{3}\left(L^{\prime}\right)=s_{3}(L)$. Thus, $|X|$ is equal to 56 or 280 .

Let $|X|=56$; then $n_{0}=n_{2}=0$ by the equality (10). Since $p_{0}>0$ and $p_{1}>0$, we can take some elements $x 1, x_{2} \in X$ such that $\left(x_{1}, t\right)=0,\left(x_{2}, t\right)=1$, and $\left(x_{1}, x_{2}\right)= \pm 1$. Then, $x_{1} \mp\left(x_{2}-t\right)$ is a vector of norm 3, i.e. $x_{1} \mp\left(x_{2}-t\right) \in X$, and we have $\left(x_{1} \mp\left(x_{2}-t\right), x_{1}\right)=2$. This contradicts $n_{2}=0$.

Let $|X|=280$; then we have $L \supset L^{\prime} \simeq \mathbb{Z}^{7}$. Since $L$ is integral, we have $L \simeq \mathbb{Z}^{7}$.

In conclusion, if $\min (L)=1$, then we have $L \simeq \mathbb{Z}^{7}$.

Now, the remaining case is when $\min (L)=2$.

If $\min (L)=2$, by the equations (10) and (12), we have the following equations:

$$
\begin{aligned}
n=16, \quad p_{0} & =\frac{5}{8}|X|, \quad p_{1}=\frac{3}{16}|X| . \\
n_{0}=\frac{65}{128}|X|-20, \quad n_{1} & =\frac{15}{64}|X|+15, \quad n_{2}=\frac{3}{256}|X|-6 .
\end{aligned}
$$

Since $n_{i}$ and $p_{i}$ are nonnegative integers,

$$
256|| X \mid \text { and }|X| \geqslant 512
$$

Furthermore, for any $x_{0} \in X$, if $x \in X$ satisfies $\left(x_{0}, x\right)=2$, then $x_{0}-x \in s_{2}(L)$ and $\left(x_{0}-x, x_{0}\right)=1$. On the other hand, if $y \in s_{2}(L)$ satisfies $\left(x_{0}, y\right)=1$, then $x_{0}-y \in X$ and $\left(x_{0}-y, x_{0}\right)=2$. Thus, we have

$$
\left|\left\{y \in s_{2}(L) ;\left(x_{0}, y\right)=1\right\}\right|=\left|\left\{x \in X ;\left(x_{0}, x\right)=2\right\}\right|=n_{2},
$$

where this number does not depend on the choice of $x_{0}$. Then, we have

$$
\begin{aligned}
n_{2}|X| & =\left|\left\{y \in s_{2}(L) ;\left(x_{0}, y\right)=1\right\}\right| \times|X| \\
& =\left|\left\{x \in X ;\left(x, y_{0}\right)=1\right\}\right| \times\left|s_{2}(L)\right|=p_{1}\left|s_{2}(L)\right| .
\end{aligned}
$$


Thus, we have

$$
\left|s_{2}(L)\right|=\frac{1}{16}|X|-32 .
$$

\section{INTERSECTION NUMBERS}

Let $\alpha, \beta, \gamma \in A(X)$, where $A(X)=\{(x, y) ; x, y \in X, x \neq y\}$ is a distance set. Then, we choose a pair of vectors $x, y \in X$ such that $(x, y)=\gamma$, and denote

$$
P_{\gamma}(\alpha, \beta):=|\{z \in X ;(x, z)=\alpha,(z, y)=\beta\}| .
$$

If this number is uniquely determined for any choice of the pair $x, y$, then it can be called the intersection number. Since $X$ is antipodal, we have $P_{\gamma}(\alpha, \beta)=P_{\gamma}(\beta, \alpha)$ and $P_{\gamma}(\alpha, \beta)=P_{\gamma}(-\alpha,-\beta)=P_{-\gamma}(\alpha,-\beta)$.

In this section, the intersection numbers $P_{2}(\alpha, \beta)$ are considered. Now, a pair of vectors $x, y \in X$ such that $(x, y)=2$ is chosen. Then, we have $x-y \in s_{2}(L)$. For any $z \in X$, we have $(x-y, z)=(x, z)-(y, z) \in$ $\{0, \pm 1\}$ by the relation (11). Here, we have $P_{2}(\alpha, \beta)=0$ for every $\alpha, \beta$ such that $|\alpha-\beta|>1$. Furthermore, it is clear that $P_{2}(3,3)=0$ and $P_{2}(2,3)=1$. This can be denoted

$$
a_{1}:=P_{2}(2,2), \quad a_{2}:=P_{2}(1,2), \quad a_{3}:=P_{2}(1,1), \quad a_{4}:=P_{2}(0,1), \quad a_{5}:=P_{2}(0,0) .
$$

We have

$$
n_{0}=2 a_{4}+a_{5}, \quad n_{1}=a_{2}+a_{3}+a_{4}, \quad n_{2}=1+a_{1}+a_{2} .
$$

By the equations (8) and (9), taking $\alpha=x+y$ and $\alpha=x-y$, we obtain four relations for $a_{1}, \ldots, a_{5}$. Finally, we have

$$
\begin{gathered}
P_{2}(2,2)=\frac{1}{256}|X|-4, \quad P_{2}(1,2)=\frac{1}{128}|X|-3, \quad P_{2}(1,1)=\frac{9}{64}|X|+16, \\
P_{2}(0,1)=\frac{11}{128}|X|+2, \quad P_{2}(0,0)=\frac{43}{128}|X|-24 .
\end{gathered}
$$

Now, for any element $y_{0} \in s_{2}(L)$, there exists $x_{0} \in X$ such that $\left(x_{0}, y_{0}\right)=1$. Then, we have $x_{0}-y_{0} \in X$ and $\left(x_{0}-y_{0}, x_{0}\right)=2$. Let $y \in s_{2}(L)$ satisfy $\left(y_{0}, y\right)=1$, then $y_{0}-y \in s_{2}(L)$ and $\left(x_{0}, y\right)=0$ or 1 , because $\left(x_{0}, y_{0}-y\right)=1-\left(x_{0}, y\right) \in\{0, \pm 1\}$ by $(11)$. If $\left(x_{0}, y\right)=1$, then $x_{0}-y \in X$ and $\left(x_{0}, x_{0}-y\right)=\left(x_{0}-y_{0}, x_{0}-y\right)=2$. Thus, we have

$$
\left|\left\{y \in s_{2}(L) ;\left(y_{0}, y\right)=1,\left(x_{0}, y\right)=1\right\}\right|=\left|\left\{x \in X ;\left(x_{0}, x\right)=\left(x_{0}-y_{0}, x\right)=2\right\}\right|=P_{2}(2,2) .
$$

On the other hand, if $\left(x_{0}, y\right)=0$, then $y_{0}-y \in X$ and $\left(y_{0}, y_{0}-y\right)=\left(x_{0}, y_{0}-y\right)=1$. Thus, we have

$$
\left|\left\{y \in s_{2}(L) ;\left(y_{0}, y\right)=1,\left(x_{0}, y\right)=0\right\}\right|=\left|\left\{y \in s_{2}(L) ;\left(y_{0}, y\right)=1,\left(x_{0}, y\right)=1\right\}\right|=P_{2}(2,2) .
$$

In conclusion, we have

$$
\left|\left\{y \in s_{2}(L) ;\left(y_{0}, y\right)=1\right\}\right|=\frac{1}{128}|X|-8, \quad\left|\left\{y \in s_{2}(L) ;\left(y_{0}, y\right)=0\right\}\right|=\frac{1}{64}|X|-18 .
$$

These relations imply that $s_{2}(L)$ is a spherical 3-design.

In addition, we have the following fact:

Lemma 4.1 (see [7]). Let $L$ be an integral lattice. Then, its shell $s_{2}(L)$ of norm 2 is a root system.

Furthermore, irreducible root systems have been classified; they are $A_{n}$ for $n \geqslant 1, D_{n}$ for $n \geqslant 4$, and $E_{n}$ for $n=6,7,8$ (see [1]). Orthogonal unions of irreducible root systems which satisfy the conditions (15), (17) and (19) result only in the following nine cases:

$$
s_{2}(L) \simeq\left(A_{1}\right)^{16},\left(A_{2}\right)^{8},\left(A_{4}\right)^{4},\left(A_{8}\right)^{2}, A_{16},\left(D_{4}\right)^{4},\left(D_{8}\right)^{2}, D_{16} \text {, or }\left(E_{8}\right)^{2} .
$$

In the next section, each case is examined. Finally, only the three cases $s_{2}(L)=\left(A_{1}\right)^{16},\left(D_{4}\right)^{4}$, and $\left(D_{8}\right)^{2}$ will have to be considered, and obtain lattices $\Lambda_{16,2,1}, \Lambda_{16,2,2}$, and $\Lambda_{16,2,3}$, respectively. 


\section{Classification of lattices}

5.1. The case of $s_{2}(L)=\left(A_{1}\right)^{16}$.

We put $\left(A_{1}\right)^{16}=\left\{ \pm \sqrt{2} e_{i} ; 1 \leqslant i \leqslant 16\right\}$ with an orthonormal basis $\left\{e_{i}\right\}_{1 \leqslant i \leqslant 16}$ of $\mathbb{R}^{16}$. Consider a vector $x_{0} \in X$. We write $x_{0}=\left(a_{1}, \ldots, a_{16}\right) \in X$, then we have $\left(x, \sqrt{2} e_{i}\right)=\sqrt{2} a_{i} \in\{0, \pm 1\}$, thus we have $a_{i} \in\{0, \pm 1 / \sqrt{2}\}$. In addition, we have $(x, x)=a_{1}^{2}+\cdots+a_{16}^{2}=3$ by definition. Thus, six coordinates are $\pm 1 \sqrt{2}$ and ten coordinates are 0 .

If $a_{i}=1 / \sqrt{2}$, then the $i$ th coordinate of $x_{0}-\sqrt{2} e_{i}$ is equal to $-1 / \sqrt{2}$. Thus, in the lattice which is an additional group, we take all possible sign changes of nonzero coordinates of $x_{0}$. We write $\overline{x_{0}}=$ $\sqrt{2}\left(\left|a_{1}\right|, \ldots,\left|a_{16}\right|\right)$, then we define equivalence classes $\bar{X}:=\{\bar{x} ; x \in X\}$, where we regard $\bar{x}$ as a equivalence class. Each class has $2^{6}$ vectors by sign changes. Since $|X|=1024$, we have 16 classes.

Take $x_{1} \notin \overline{x_{0}}$, and let $l:=\left(\overline{x_{0}}, \overline{x_{1}}\right)$. In this case, we have $n_{0}=500, n_{1}=255$, and $n_{2}=6$. Firstly, since $\left(x_{1}, x_{0}\right) \in\{0, \pm 1, \pm 2\}$, we have $l=0,2,4$. We write $m_{i}{ }^{\prime}:=\left|\left\{x \in \overline{x_{0}} ;\left(x, x_{0}\right)=i\right\}\right|$ and $m_{l, i}{ }^{\prime \prime}:=\left|\left\{x \in \overline{x_{1}} ;\left(x, x_{0}\right)=i\right\}\right|$ for $i=0,1,2$. Then, we have $m_{0}{ }^{\prime}=20, m_{1}{ }^{\prime}=15$, and $m_{2}{ }^{\prime}=6$. Secondly, since $m_{2}^{\prime}=n_{2}$, we need $m_{l, 2}{ }^{\prime \prime}=0$, thus we have $l=0,2$. Finally, we have $m_{0,0}{ }^{\prime \prime}=64, m_{2,0}{ }^{\prime \prime}=32$, and $n_{0}=20+32 \times 15$ for 16 equivalent classes, so we have $l=2$.

Now, if we regard $\bar{X}$ as an incidence matrix of a block design (cf. matrix (21)), we can consider a 2-(16,6,2) $(t-(v, k, \lambda))$ design. By Gibbons [4], we have just three equivalence classes for this block design. Furthermore, if we regard the three classes as a basis for a linear code of $\mathbb{F}_{2}^{16}$, then we obtain three linear codes whose parameters are respectively $[v, k, d]=[16,6,6],[16,7,4]$, and $[16,8,4]$. Moreover, when $[v, k, d]=[16,7,4]$ and $[16,8,4]$, we have more than 16 code words of length 6 which correspond to the equivalence classes of $\bar{X}$. For example, the following is an incident matrix of a 2-(16,6,2) block design from which we obtain $[16,6,6]$-linear code of $\mathbb{F}_{2}^{16}$ :

$$
\left[\begin{array}{llllllllllllllll}
1 & 1 & 0 & 0 & 0 & 0 & 0 & 0 & 1 & 0 & 1 & 0 & 1 & 0 & 1 & 0 \\
1 & 1 & 0 & 0 & 0 & 0 & 0 & 0 & 0 & 1 & 0 & 1 & 0 & 1 & 0 & 1 \\
1 & 0 & 1 & 0 & 1 & 0 & 1 & 0 & 1 & 1 & 0 & 0 & 0 & 0 & 0 & 0 \\
1 & 0 & 1 & 0 & 0 & 1 & 0 & 1 & 0 & 0 & 1 & 1 & 0 & 0 & 0 & 0 \\
1 & 0 & 0 & 1 & 1 & 0 & 0 & 1 & 0 & 0 & 0 & 0 & 1 & 1 & 0 & 0 \\
1 & 0 & 0 & 1 & 0 & 1 & 1 & 0 & 0 & 0 & 0 & 0 & 0 & 0 & 1 & 1 \\
0 & 1 & 1 & 0 & 1 & 0 & 0 & 1 & 0 & 0 & 0 & 0 & 0 & 0 & 1 & 1 \\
0 & 1 & 1 & 0 & 0 & 1 & 1 & 0 & 0 & 0 & 0 & 0 & 1 & 1 & 0 & 0 \\
0 & 1 & 0 & 1 & 1 & 0 & 1 & 0 & 0 & 0 & 1 & 1 & 0 & 0 & 0 & 0 \\
0 & 1 & 0 & 1 & 0 & 1 & 0 & 1 & 1 & 1 & 0 & 0 & 0 & 0 & 0 & 0 \\
0 & 0 & 1 & 1 & 0 & 0 & 0 & 0 & 1 & 0 & 1 & 0 & 0 & 1 & 0 & 1 \\
0 & 0 & 1 & 1 & 0 & 0 & 0 & 0 & 0 & 1 & 0 & 1 & 1 & 0 & 1 & 0 \\
0 & 0 & 0 & 0 & 1 & 1 & 0 & 0 & 1 & 0 & 0 & 1 & 1 & 0 & 0 & 1 \\
0 & 0 & 0 & 0 & 1 & 1 & 0 & 0 & 0 & 1 & 1 & 0 & 0 & 1 & 1 & 0 \\
0 & 0 & 0 & 0 & 0 & 0 & 1 & 1 & 1 & 0 & 0 & 1 & 0 & 1 & 1 & 0 \\
0 & 0 & 0 & 0 & 0 & 0 & 1 & 1 & 0 & 1 & 1 & 0 & 1 & 0 & 0 & 1
\end{array}\right] .
$$

Thus, we can determine the lattices $L$ for which $s_{3}(L)$ is spherical 5-design and $s_{2}(L)=\left(A_{1}\right)^{16}$ uniquely up to isometry.

Now, let $\left\{\varepsilon_{i}\right\}_{1 \leqslant i \leqslant 16}$ be another orthonormal basis of $\mathbb{R}^{16}$, and take an isometry which maps

$$
\sqrt{2} e_{2 i-1} \mapsto \varepsilon_{2 i-1}+\varepsilon_{2 i} \quad \text { and } \quad \sqrt{2} e_{2 i} \mapsto \varepsilon_{2 i-1}-\varepsilon_{2 i} \quad \text { for every } 1 \leqslant i \leqslant 8 .
$$

Then, this isometry leads the definition of $\Lambda_{16,2,1}$ in Section 1 from the above lattice which corresponds to the matrix (21). Actually, from the first and fourth row of the above matrix, we have the following correspondences:

$$
\begin{aligned}
\frac{e_{1}+e_{2}+e_{9}+e_{11}+e_{13}+e_{15}}{\sqrt{2}} & \mapsto f_{2}=\varepsilon_{1}+\frac{\varepsilon_{9}+\cdots+\varepsilon_{16}}{2}, \\
\frac{e_{1}+e_{3}+e_{5}+e_{7}+e_{9}+e_{10}}{\sqrt{2}} & \mapsto f_{1}=\frac{\varepsilon_{1}+\cdots+\varepsilon_{8}}{2}+\varepsilon_{9} .
\end{aligned}
$$

5.2. The cases of $s_{2}(L)=\left(A_{2}\right)^{8},\left(A_{4}\right)^{4},\left(A_{8}\right)^{2}, A_{16}$.

We can write $A_{n}=\left\{ \pm \varepsilon_{i} \mp \varepsilon_{j} ; 1 \leqslant i<j \leqslant n+1\right\}$. We take $x_{0} \in X$. If there exists $y \in A_{n}$ such that $\left(x_{0}, y\right)=1$, then we may assume $y=\varepsilon_{1}-\varepsilon_{2}$ without loss of generality. Furthermore, we write $m_{1}{ }^{\prime}:=\left|\left\{y \in A_{n} ;\left(x_{0}, y\right)=1\right\}\right|$. Now, we consider several distinct cases, namely $(i) y= \pm\left(\varepsilon_{1}-\varepsilon_{2}\right),(i i)$ $y= \pm\left(\varepsilon_{1}-\varepsilon_{i}\right), \pm\left(\varepsilon_{2}-\varepsilon_{i}\right)$ for $3 \leqslant i \leqslant n+1$, and $(i i i) y= \pm\left(\varepsilon_{i}-\varepsilon_{j}\right)$ for $3 \leqslant i<j \leqslant n+1$. 
For the second case $(i i)$, we have $\left(x_{0}, \varepsilon_{1}-\varepsilon_{i}\right)-\left(x_{0}, \varepsilon_{2}-\varepsilon_{i}\right)=\left(x_{0}, \varepsilon_{1}-\varepsilon_{2}\right)=1$. If we have $\left(x_{0}, \varepsilon_{1}-\right.$ $\left.\varepsilon_{i}\right)=-1$, then $\left(x_{0}, \varepsilon_{2}-\varepsilon_{i}\right)=-2$, contradicting the relation (11). Thus, $\left(x_{0}, \varepsilon_{1}-\varepsilon_{i}\right)=0,1$ and $\left(x_{0}, \varepsilon_{2}-\varepsilon_{i}\right)=0,-1$; just one of $\left(x_{0}, \varepsilon_{1}-\varepsilon_{i}\right)$ and $\left(x_{0}, \varepsilon_{2}-\varepsilon_{i}\right)$ is nonzero. Moreover, half of the vectors of this case are orthogonal to $x_{0}$.

For the third case $(i i i)$, we have $\left(x_{0}, \varepsilon_{i}-\varepsilon_{j}\right)=-\left(x_{0}, \varepsilon_{1}-\varepsilon_{i}\right)+\left(x_{0}, \varepsilon_{1}-\varepsilon_{j}\right) .\left(x_{0}, \varepsilon_{i}-\varepsilon_{j}\right)$ is nonzero if and only if just one of $\left(x_{0}, \varepsilon_{1}-\varepsilon_{i}\right)$ and $\left(x_{0}, \varepsilon_{1}-\varepsilon_{j}\right)$ is equal to 1 . We put $D:=\left|\left\{3 \leqslant i \leqslant n+1 ;\left(x_{0}, \varepsilon_{1}-\varepsilon_{i}\right)=1\right\}\right|$, then $2 D(n-1-D)$ of vectors of this case are not orthogonal to $x_{0}$.

In conclusion, we have $m_{1}{ }^{\prime}=1+(n-1)+D(n-1-D)=(n-D)(D+1)$. Note that $m_{1}{ }^{\prime}$ is even if $n$ is even.

Recall that $\left|\left\{y \in s_{2}(L) ;\left(x_{0}, y\right)=1\right\}\right|=n_{2}$, and note that this number for $s_{2}(L)$ must be a combination of the numbers $m_{1}{ }^{\prime}$ for each $A_{n}$. However, if $n=2,4,8,16$, then we have $n_{2}=9,15,27,51$, where all of them are odd. These facts are contradictory. Thus, we can omit these cases, when $s_{2}(L)=\left(A_{n}\right)^{16 / n}$ for $n>1$.

5.3. The cases of $s_{2}(L)=\left(D_{4}\right)^{4},\left(D_{8}\right)^{2}, D_{16}$.

Following the procedure of the previous section, we write $D_{n}=\left\{ \pm \varepsilon_{i} \pm \varepsilon_{j}, \pm \varepsilon_{i} \mp \varepsilon_{j} ; 1 \leqslant i<j \leqslant n\right\}$ for an orthonormal basis of $\mathbb{R}^{n}$. We take $x_{0} \in X$, then we assume $\left(x_{0}, \varepsilon_{1}+\varepsilon_{2}\right)=1$ if some vector of $D_{n}$ is not orthogonal to $x_{0}$. Furthermore, we write $m_{1}{ }^{\prime}:=\left|\left\{y \in D_{n} ;\left(x_{0}, y\right)=1\right\}\right|$. Now, we consider several distinct cases, namely $(i) y= \pm\left(\varepsilon_{1}+\varepsilon_{2}\right)$, (ii) $y= \pm\left(\varepsilon_{1} \pm \varepsilon_{i}\right), \pm\left(\varepsilon_{2} \pm \varepsilon_{i}\right)$ for $3 \leqslant i \leqslant n,(i i i) y= \pm\left(\varepsilon_{i} \pm \varepsilon_{j}\right)$ for $3 \leqslant i<j \leqslant n$, and (iv) $y= \pm\left(\varepsilon_{1}-\varepsilon_{2}\right)$.

For the second case $(i i)$, we have $\left(x_{0}, \varepsilon_{1} \pm \varepsilon_{i}\right)+\left(x_{0}, \varepsilon_{2} \mp \varepsilon_{i}\right)=1$. As in the section above, half of the vectors of this case are orthogonal to $x_{0}$.

For the fourth case $(i v)$, we put $D:=\left(x_{0}, \varepsilon_{1}+\varepsilon_{i}\right)+\left(x_{0}, \varepsilon_{1}-\varepsilon_{i}\right)=\left(x_{0}, 2 \varepsilon_{1}\right)$ for any $3 \leqslant i \leqslant n$, which does not depend on $i$. If $D=0$, then $\left(x_{0}, \varepsilon_{2}+\varepsilon_{i}\right)+\left(x_{0}, \varepsilon_{2}-\varepsilon_{i}\right)=1$; thus we can take $\varepsilon_{2}$ instead of $\varepsilon_{1}$ without loss of generality. We may assume $D=1,2$.

If $D=1$, then $\left(x_{0}, \varepsilon_{1}-\varepsilon_{2}\right)=0$. For the third case $(i i i)$, we have $\left(x_{0}, \varepsilon_{i} \pm \varepsilon_{j}\right)=\left(x_{0}, \varepsilon_{1}+\varepsilon_{i}\right)-\left(x_{0}, \varepsilon_{1} \pm \varepsilon_{j}\right)$. Thus, just one of $\left(x_{0}, \varepsilon_{i}+\varepsilon_{j}\right)$ and $\left(x_{0}, \varepsilon_{i}-\varepsilon_{j}\right)$ is zero. Moreover, half of the vectors of the case $($ iii $)$ are orthogonal to $x_{0}$. In conclusion, we have $m_{1}^{\prime}=1+2(n-2)+(n-2)(n-3) / 2+0=n(n-1) / 2$.

If $D=2$, then $\left(x_{0}, \varepsilon_{1}-\varepsilon_{2}\right)=1$. For the third case $(i i i)$, we have $\left(x_{0}, \varepsilon_{i} \pm \varepsilon_{j}\right)=\left(x_{0}, \varepsilon_{1}+\varepsilon_{i}\right)-$ $\left(x_{0}, \varepsilon_{1} \pm \varepsilon_{j}\right)=0$. Then, all of the vectors of the case $(i i i)$ are orthogonal to $x_{0}$. In conclusion, we have $m_{1}^{\prime}=1+2(n-2)+0+1=2(n-1)$.

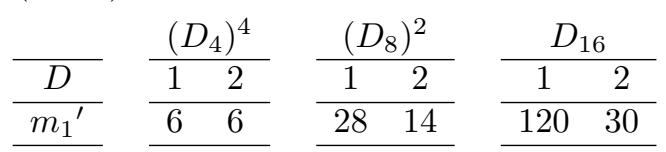

Recall that $\left|\left\{y \in s_{2}(L) ;\left(x_{0}, y\right)=1\right\}\right|=n_{2}$, and note that this number must be the sum of the numbers $m_{1}{ }^{\prime}$. If $n=4,8,16$, then we have $n_{2}=18,42,90$, respectively. Then, there remain some possibilities such that $n_{2}=6+6+6$ for the case of $s_{2}(L)=\left(D_{4}\right)^{4}$ and $n_{2}=28+14$ for the case of $s_{2}(L)=\left(D_{8}\right)^{2}$. On the other hand, $n_{2} \neq m_{1}{ }^{\prime}$ for the case of $s_{2}(L)=D_{16}$; thus we can omit this case.

Now, we write $x_{0}=\left(a_{1}, \ldots, a_{n}, a_{n+1}, \ldots, a_{16}\right)$, where each $a_{i}$ for $1 \leqslant i \leqslant n$ is the coordinate of $\varepsilon_{i}$ used for $D_{n}$. Then, we have $\left(x_{0}, \varepsilon_{i} \pm \varepsilon_{j}\right)=a_{i} \pm a_{j}$, thus we can calculate each $a_{i}$ from the values of $\left(x_{0}, \varepsilon_{i} \pm \varepsilon_{j}\right)$.

If $D=1$, then we have $\left|a_{i}\right|=\frac{1}{2}$ for every $1 \leqslant i \leqslant n$. Furthermore, for $2 a_{i} \varepsilon_{i}+2 a_{j} \varepsilon_{j} \in D_{n}$, $x_{0}-\left(2 a_{i} \varepsilon_{i}+2 a_{j} \varepsilon_{j}\right)$ is also a vector of norm 3 , where both signs of the $i$ th and $j$ th coordinates are different from those of $x_{0}$. Similarly, we have every element whose even signs are different from $x_{0}$. Here, we write

$$
\begin{aligned}
& L_{2}:=\left\{( \pm 1, \ldots, \pm 1) / 2 \in \mathbb{R}^{n} ; \text { the number of "-" is even }\right\}, \\
& L_{3}:=\left\{( \pm 1, \ldots, \pm 1) / 2 \in \mathbb{R}^{n} ; \text { the number of "-_" is odd }\right\}
\end{aligned}
$$

then the lattice $L$ includes either $L_{2} \times\left\{\left(a_{n+1}, \ldots, a_{16}\right)\right\}$ or $L_{3} \times\left\{\left(a_{n+1}, \ldots, a_{16}\right)\right\}$.

If $D=2$, then we have $\left|a_{i}\right|=1$ for some $1 \leqslant i \leqslant n$ and $a_{j}=0$ for every $1 \leqslant j \leqslant n$ such that $i \neq j$. Furthermore, for $2 a_{i} \varepsilon_{i}$ and $a_{i} \varepsilon_{i} \pm \varepsilon_{j} \in D_{n}, x_{0}-2 a_{i} \varepsilon_{i}$ and $x_{0}-\left(a_{i} \varepsilon_{i} \pm \varepsilon_{j}\right)$ is also a vector of norm 3 . Here, we write

$$
L_{1}:=\left\{ \pm \varepsilon_{1}, \ldots, \pm \varepsilon_{n}\right\},
$$

then the lattice $L$ includes $L_{1} \times\left\{\left(a_{n+1}, \ldots, a_{16}\right)\right\}$. 
On the other hand, if $x_{0}$ is orthogonal to $D_{n}$, then $a_{i}=0$ for every $1 \leqslant i \leqslant n$. Here, we write

$$
L_{0}:=\left\{(0, \ldots, 0) \in \mathbb{R}^{n}\right\}
$$

then we can say that the lattice $L$ includes $L_{0} \times\left\{\left(a_{n+1}, \ldots, a_{16}\right)\right\}$.

5.3.1. The case of $s_{2}(L)=\left(D_{4}\right)^{4}$. We have $L_{1} \simeq L_{2} \simeq L_{3} \simeq \mathbb{Z}^{4}$, where each vector is of norm 1. Thus, $s_{3}(L) \supset L_{i_{1}} \times L_{i_{2}} \times L_{i_{3}} \times L_{i_{4}}$, where $i_{k}=0$ for just one $k$ and this set has 512 vectors. Since $\left|s_{3}(L)\right|=2048$, $s_{3}(L)$ includes four such sets. Then, we write

$$
\begin{array}{r}
L_{I}=\left(L_{i_{1,1}} \times L_{i_{1,2}} \times L_{i_{1,3}} \times L_{i_{1,4}}\right) \cup\left(L_{i_{2,1}} \times L_{i_{2,2}} \times L_{i_{2,3}} \times L_{i_{2,4}}\right) \\
\cup\left(L_{i_{3,1}} \times L_{i_{3,2}} \times L_{i_{3,3}} \times L_{i_{3,4}}\right) \cup\left(L_{i_{4,1}} \times L_{i_{4,2}} \times L_{i_{4,3}} \times L_{i_{4,4}}\right),
\end{array}
$$

where $I=\left(i_{1,1}, \ldots, i_{4,4}\right)$.

Firstly, for every $y_{0} \in D_{4}$, we have $p_{1}=384$ and $\left|\left\{x \in L_{i_{j, 1}} \times L_{i_{j, 2}} \times L_{i_{j, 3}} \times L_{i_{j, 4}} ;\left(x, y_{0}\right)=1\right\}\right|=0$ or 128. Thus, we have that just one of $i_{1, k}, i_{2, k}, i_{3, k}, i_{4, k}$ is zero for each $1 \leqslant k \leqslant 4$. We may assume that $i_{1,4}=i_{2,3}=i_{3,2}=i_{4,1}=0$, without loss of generality. Secondly, we consider the inner product $\left(x_{1}, x_{2}\right)$ for $x_{1} \in L_{i_{k}}$ and $x_{2} \in L_{j_{k}}$. We have the fact that $\left(x_{1}, x_{2}\right)=0, \pm 1$ if $i_{k}=j_{k} \neq 0$, that $\left(x_{1}, x_{2}\right)= \pm 1 / 2$ if $i_{k} \neq j_{k}$ and $i_{k} \cdot j_{k} \neq 0$, and that $\left(x_{1}, x_{2}\right)=0$ if $i_{k} \cdot j_{k}=0$. Thus, we have $i_{j_{1}, k} \neq i_{j_{2}, k}$ for $1 \leqslant k \leqslant 4$ if $j_{1} \neq j_{2}$. Furthermore, $\left\{i_{1, k}, i_{2, k}, i_{3, k}, i_{4, k}\right\}=\{0,1,2,3\}$ for $1 \leqslant k \leqslant 4$. Finally, there is an isometry which maps $L_{i}$ to $L_{i^{\prime}}$ for every $1 \leqslant i \leqslant 4$ and some $1 \leqslant i^{\prime} \leqslant 4$. Thus, for every pair $I=\left(i_{1,1}, \ldots, i_{4,4}\right)$ which satisfies $i_{1,4}=i_{2,3}=i_{3,2}=i_{4,1}=0$ and $\left\{i_{1, k}, i_{2, k}, i_{3, k}, i_{4, k}\right\}=\{0,1,2,3\}$ for each $1 \leqslant k \leqslant 4, L_{I}$ is isometric to the following set:

$$
\begin{aligned}
& \left(L_{2} \times L_{2} \times L_{1} \times L_{0}\right) \cup\left(L_{3} \times L_{3} \times L_{0} \times L_{1}\right) \\
& \quad \cup\left(L_{1} \times L_{0} \times L_{2} \times L_{2}\right) \cup\left(L_{0} \times L_{1} \times L_{3} \times L_{3}\right) \\
& \subset\left(f_{1}+\left(D_{4}\right)^{4}\right) \cup\left(f_{1}-f_{3}+\left(D_{4}\right)^{4}\right) \\
& \cup\left(f_{2}+\left(D_{4}\right)^{4}\right) \cup\left(f_{2}-f_{3}+\left(D_{4}\right)^{4}\right) .
\end{aligned}
$$

Thus, we can determine $s_{3}(L)$ uniquely up to isometry, which generates $\Lambda_{16,2,2}$ in Section 1 .

5.3.2. The case of $s_{2}(L)=\left(D_{8}\right)^{2}$. Every vector of $L_{1}$ is of norm 1 , and every vector of $L_{2}$ and $L_{3}$ is of norm 2. Thus, $s_{3}(L) \supset L_{1} \times L_{i}$ or $L_{j} \times L_{1}$ for some $i, j=2,3$, where each set has 2048 vectors. Similar to the previous section, we have

$$
L_{i, j}=\left(L_{1} \times L_{i}\right) \cup\left(L_{j} \times L_{1}\right) .
$$

When we consider an isometry which changes the sign of one fixed coordinate of each vector, then this maps $L_{2}$ to $L_{3}$ and stabilizes $L_{1}$. Thus, for every $i, j=2,3, L_{i, j}$ is isometric to the following set:

$$
\left(L_{2} \times L_{1}\right) \cup\left(L_{1} \times L_{2}\right) \subset\left(f_{1}+\left(D_{8}\right)^{2}\right) \cup\left(f_{2}+\left(D_{8}\right)^{2}\right)
$$

Thus we can determine $s_{3}(L)$ uniquely up to isometry, which generates $\Lambda_{16,2,3}$ in Section 1 .

5.4. The case of $s_{2}(L)=\left(E_{8}\right)^{2}$. Similar to the case of $s_{2}(L)=\left(D_{8}\right)^{2}$, we write $E_{8}=\left\{ \pm \varepsilon_{i} \pm \varepsilon_{j}, \pm \varepsilon_{i} \mp\right.$ $\left.\varepsilon_{j} ; 1 \leqslant i<j \leqslant 8\right\} \cup\left\{\left( \pm \varepsilon_{1} \pm \cdots \pm \varepsilon_{8}\right) / 2\right.$; the number of '- ' is even $\}$ for an orthonormal basis of $\mathbb{R}^{8}$. We take $x_{0} \in X$, then we assume that $\left(x_{0}, \varepsilon_{1}+\varepsilon_{2}\right)=1$ if such an element exists. Furthermore, we write $m_{1}^{\prime}:=\left|\left\{y \in E_{8} ;\left(x_{0}, y\right)=1\right\}\right|$. We consider several distinct cases; namely $(i) y= \pm\left(\varepsilon_{1}+\varepsilon_{2}\right),(i i)$ $y= \pm\left(\varepsilon_{1} \pm \varepsilon_{i}\right), \pm\left(\varepsilon_{2} \pm \varepsilon_{i}\right)$ for $3 \leqslant i \leqslant 8$, (iii) $y= \pm\left(\varepsilon_{i} \pm \varepsilon_{j}\right)$ for $3 \leqslant i<j \leqslant 8$, (iv) $y= \pm\left(\varepsilon_{1}-\varepsilon_{2}\right),(v)$ $y= \pm\left(\varepsilon_{1}+\varepsilon_{2} \pm \varepsilon_{3} \pm \cdots \pm \varepsilon_{8}\right) / 2$, and $(v i) y= \pm\left(\varepsilon_{1}-\varepsilon_{2} \pm \varepsilon_{3} \pm \cdots \pm \varepsilon_{8}\right) / 2$.

The cases $(i),(i i),(i i i)$, and $(v i)$ are similar to the case of $s_{2}(L)=\left(D_{8}\right)^{2}$. Note that $\left(x_{0}, \varepsilon_{1} \pm \varepsilon_{j}\right)=0,1$ and $\left(x_{0}, \varepsilon_{2} \pm \varepsilon_{j}\right)=0,1$, and we put $D:=\left(x_{0}, \varepsilon_{1}+\varepsilon_{i}\right)+\left(x_{0}, \varepsilon_{1}-\varepsilon_{i}\right) \in\{1,2\}$.

For the fifth case $(v)$, we have $\left(x_{0}, \varepsilon_{1}+\varepsilon_{2}\right)=\left(x_{0},\left(\varepsilon_{1}+\varepsilon_{2} \pm \varepsilon_{3} \pm \cdots \pm \varepsilon_{8}\right) / 2\right)+\left(x_{0},\left(\varepsilon_{1}+\varepsilon_{2} \mp \varepsilon_{3} \mp \cdots \mp \varepsilon_{8}\right) / 2\right)$. Thus, half of the vectors of this case are orthogonal to $x_{0}$.

If $D=2$, we have $\left(x_{0}, \varepsilon_{1}-\varepsilon_{2}\right)=1$. The sixth case $(v i)$ is similar to the case $(v)$, and half of the vectors of this case are orthogonal to $x_{0}$. We have $m_{1}{ }^{\prime}=1+12+0+1+16+16=46$.

If $D=1$, just one of $\left(x_{0}, \varepsilon_{i}+\varepsilon_{j}\right)$ and $\left(x_{0}, \varepsilon_{i}-\varepsilon_{j}\right)$ is zero for the case $(i i i)$. We may assume $\left(x_{0}, \varepsilon_{3}+\varepsilon_{4}\right)=$ $\left(x_{0}, \varepsilon_{5}+\varepsilon_{6}\right)=\left(x_{0}, \varepsilon_{7}+\varepsilon_{8}\right)=1$ without loss of generality. Firstly, when the sign of $\varepsilon_{3}$ is equal to that of $\varepsilon_{4}$, then we have $\left(x_{0}, \varepsilon_{3}+\varepsilon_{4}\right)=\left(x_{0},\left( \pm \varepsilon_{1} \mp \varepsilon_{2}+\varepsilon_{3}+\varepsilon_{4} \pm \varepsilon_{5} \pm \cdots \pm \varepsilon_{8}\right) / 2\right)-\left(x_{0},\left( \pm \varepsilon_{1} \mp \varepsilon_{2}-\varepsilon_{3}-\varepsilon_{4} \pm \varepsilon_{5} \pm \cdots \pm \varepsilon_{8}\right) / 2\right)$; thus half of such vectors are orthogonal to $x_{0}$. Secondly, when 'the sign of $\varepsilon_{3}$ is not equal to that of $\varepsilon_{4}$ ' and 'the sign of $\varepsilon_{5}$ is equal to that of $\varepsilon_{6}$ ', then $\left(x_{0}, \varepsilon_{5}+\varepsilon_{6}\right)=\left(x_{0},\left( \pm \varepsilon_{1} \mp \varepsilon_{2} \pm \varepsilon_{3} \mp \varepsilon_{4}+\varepsilon_{5}+\varepsilon_{6} \pm \varepsilon_{7} \mp\right.\right.$ 
$\left.\left.\varepsilon_{8}\right) / 2\right)-\left(x_{0},\left( \pm \varepsilon_{1} \mp \varepsilon_{2} \pm \varepsilon_{3} \mp \varepsilon_{4}-\varepsilon_{5}-\varepsilon_{6} \pm \varepsilon_{7} \mp \varepsilon_{8}\right) / 2\right)$, thus half of such vectors are orthogonal to $x_{0}$. Finally, when 'the sign of $\varepsilon_{3}$ is not equal to that of $\varepsilon_{4}$ ' and 'the sign of $\varepsilon_{5}$ is not equal to that of $\varepsilon_{6}$ ', then $\left(x_{0}, \varepsilon_{7}+\varepsilon_{8}\right)=\left(x_{0},\left( \pm \varepsilon_{1} \mp \varepsilon_{2} \pm \varepsilon_{3} \mp \varepsilon_{4} \pm \varepsilon_{5} \mp \varepsilon_{6}+\varepsilon_{7}+\varepsilon_{8}\right) / 2\right)-\left(x_{0},\left( \pm \varepsilon_{1} \mp \varepsilon_{2} \pm \varepsilon_{3} \mp \varepsilon_{4} \pm \varepsilon_{5} \mp \varepsilon_{6}-\varepsilon_{7}-\varepsilon_{8}\right) / 2\right)$; thus half of such vectors are orthogonal to $x_{0}$. In conclusion, we have $m_{1}{ }^{\prime}=1+12+15+0+16+16=60$.

Recall that $\left|\left\{y \in s_{2}(L) ;\left(x_{0}, y\right)=1\right\}\right|=n_{2}=90$, thus we cannot write $n_{2}$ as a sum of $m_{1}{ }^{\prime}$, so we can omit this case.

Remark 5.1. In the argument of Section 5.1, from the lattice $\Lambda_{16,2,1}$, we obtain a $2-(16,6,2)$ block design which generates $[16,6,6]$-linear code of $\mathbb{F}_{2}^{16}$. Conversely, it determines the lattice $\Lambda_{16,2,1}$ uniquely.

On the other hand, there are two more equivalence classes of $2-(16,6,2)$ block designs. Note that we obtain the lattices $\Lambda_{16,2,2}$ and $\Lambda_{16,2,3}$ from the two equivalence classes which generate $[16,7,4]$ and $[16,8,4]$-linear codes of $\mathbb{F}_{2}^{16}$, respectively.

\section{ACKNOWLEDGMENT}

Many thanks to Professor Eiichi Bannai for suggesting these problems. We thank the anonymous referee for many useful suggestions and comments.

\section{REFERENCES}

[1] N. Bourbaki, Groupes et algèbres de Lie Chap. IV-VI (French). Actualités Scientifiques et Industrielles, No. 1337. Hermann, Paris, 1968.

(Translation: Lie groups and Lie algebras, Springer-Verlag, Berlin, 2002.)

[2] J. H. Conway. and N. J. A. Sloane, Sphere Packings, Lattices and Groups. Springer-Verlag, Grundlehren No. 290 (Heidelberg), 1988. (Third Edition: 1999.)

[3] P. Delsarte, J. M. Goethals, and J. J. Seidel, Spherical codes and designs, Geometriae Dedicata, 6(1977), 363-388.

[4] P. B. Gibbons, Computing Techniques for the Construction and Analysis of Block Designs, Ph.D. Thesis, University of Toronto, Technical Report No. 92, May 1976. (We can also see [5])

[5] R. Mation and A. Rosa, 2- $(v, k, \lambda)$ designs of small order, in: The CRC handbook of combinatorial designs, 3-41, CRC Press, Boca Raton, FL, 1996. (Second Edition: pp.25-58, 2006])

[6] H. Nozaki, A note on odd unimodular Euclidean lattices, Arch. Math. 86 (2006), 226-230.

[7] C. Pache, Shells of selfdual lattices viewed as spherical designs, Internat. J. Algebra Comput., 15 (2005), No. 5-6, $1085-1127$.

[8] J. Shigezumi, On 3-lattices and spherical designs, ArXiv: 0810.4373 [math.CO]. cf. http://www2 .math.kyushu-u.ac.jp/ j.shigezumi/L3/index.html

[9] B. B. Venkov, Réseaux et "designs" sphériques (French) [Lattices and spherical designs], in: Réseaux euclidiens, designs sphériques et formes modulaires, 10-86, Monogr. Enseign. Math., 37, Enseignement Math., Geneva, 2001. [Notes by J. Martinet] 